\title{
Computerized Assessment of Superior Semicircular Canal Dehiscence Size using Advanced Morphological Imaging Operators
}

\author{
Joel S. Beckett ${ }^{1}$ Carlito Lagman ${ }^{1}$ Lawrance K. Chung ${ }^{1} \quad$ Timothy T. Bui $^{1} \quad$ Seung J. Lee ${ }^{1}$ \\ Brittany L. Voth ${ }^{1}$ Bilwaj Gaonkar ${ }^{1}$ Quinton Gopen ${ }^{2}$ Isaac Yang ${ }^{1,2}$
}

\footnotetext{
1 Department of Neurosurgery, David Geffen School of Medicine, University of California, Los Angeles, Los Angeles, California, United States

2 Department of Head and Neck Surgery, David Geffen School of Medicine, University of California, Los Angeles, Los Angeles, California, United States
}

\begin{abstract}
Address for correspondence Isaac Yang, MD, Departments of Neurosurgery and Head and Neck Surgery, David Geffen School of Medicine, University of California, Los Angeles, 300 Stein Plaza, Suite 562, 5th Floor Wasserman Building, Los Angeles, CA 90095-6901, United States (e-mail: iyang@mednet.ucla.edu).
\end{abstract}

J Neurol Surg B 2017;78:197-200.

\begin{abstract}
Superior semicircular canal dehiscence (SSCD) describes a pathological aperture at the level of the arcuate eminence. Techniques for quantifying defect size are described with most studies using two-dimensional lengths that underestimate the pathology. The objective of this study is to describe a novel method of measurement that combines manual segmentation of high-resolution computed tomography (HRCT) images of the temporal bone and a morphological skeletonization transform to calculate dehiscence volume. Images were imported into a freely available image segmentation tool: ITK-SNAP (version 3.4.0; available at: http://www.itksnap.org/) software. Coronal and sagittal planes were used to outline the dehiscence in all slices demonstrating the defect using the paintbrush tool. A morphological skeletonization transform derived a single-pixel thick representation

Keywords

- superior semicircular canal dehiscence

- SSCD

- size

- volume

- measurement of the original delineation. This "sheet" of voxels overlaid the dehiscence. Volume was calculated by counting the number of nonzero image voxels within this "sheet" and multiplying this number by the volume $\left(\mathrm{mm}^{3}\right)$ of each voxel. A total of 70 cases of SSCD were identified. Overall, mean volume was $0.88 \mathrm{~mm}^{3}$ (standard deviation: 0.57 , range: $0.11-2.27$ ). We present a novel technique for measuring SSCD, which we believe provides a more accurate representation of the pathology, and has the potential to standardize measurement of SSCD.
\end{abstract}

\section{Introduction}

Superior semicircular canal dehiscence (SSCD) is characterized by an abnormal opening at the level of the arcuate eminence of the petrous temporal bone. This pathological aperture can manifest clinically as a syndrome of sound- (Tullio phenomenon) and/or pressure-induced (Hennebert sign) vertigo, and oscillopsia. ${ }^{1-5}$ Auditory disturbances such as autophony and hearing loss are also common. ${ }^{6}$ Diagnosis of SSCD is based on clinical examination and findings on multislice temporal bone computed tomography (CT) with fine-cut $(0.5-0.6 \mathrm{~mm}$ collimation). ${ }^{5}$ Conservative treatment is often employed for patients with mild symptoms and includes trigger avoidance and anti-vertiginous medications. Surgical management proceeds via a middle fossa or transmastoid approach, but is reserved for patients with severe and disabling symptoms. ${ }^{3}$ received

September 19, 2016

accepted

October 10, 2016

published online

December 7, 2016 (c) 2017 Georg Thieme Verlag KG

Stuttgart · New York
DOI http://dx.doi.org/ 10.1055/s-0036-1594241. ISSN 2193-6331. 
The auditory and vestibular pathways represent an elegantly orchestrated sequence of mechanical and signal transduction. The normal oval and round windows are integral to the accommodation of pressure differentials created by sound waves. The dehiscence seen in SSCD produces a third mobile window, which not only allows for fluid and pressure wave diversion, but also communication with the middle fossa. ${ }^{3}$ Symptoms of the SSCD syndrome are attributed to the presence of this third mobile window. However, the development of dehiscence, and pathomechanisms underlying the clinical manifestations, have not yet been fully elucidated. Recent studies have attempted to correlate dehiscence size to clinical presentation and objective clinical tests. ${ }^{1,7-11}$ A significant association is commonly reported between dehiscence size and the air-bone gap (ABG), which is the difference between pure tone average (PTA) of bone conduction and PTA of air conduction. ${ }^{6,7,9,11}$ However, no strong relationship between PTA, cervical, and ocular vestibular-evoked myogenic potential (cVEMP and oVEMP, respectively), and presenting signs and symptoms, have been demonstrated.

Techniques for measuring size of dehiscence vary across studies and often employ rudimentary two-dimensional length or width measurements, despite the complex three-dimensional pathology of SSCD. Current high-resolution imaging and image segmentation software make it possible to quickly and accurately measure size in three dimensions. Herein, we describe a novel method of measuring dehiscence volume using manual segmentation of CT of the temporal bones with the freely available ITKSNAP $^{12}$ (version 3.4.0; available at: http://www.itksnap.org/() ${ }^{13}$ software. Moving forward, similar techniques may be used to more accurately represent the pathology and perhaps identify correlates that have not yet been described.

\section{Methods}

The institutional review board (IRB15-000252) approved this study. Due to the minimal risk and nature of this study, patient consent was not required (as per the IRB/ethic committee). A retrospective chart review of patients with SSCD was performed. Baseline patient characteristics were extracted from electronic medical records. Dedicated temporal bone $0.6 \mathrm{~mm} /$ slice CT images for all patients were obtained and imported into the freely available ITK-SNAP ${ }^{12}$ (version 3.4.0) ${ }^{13}$ software. Data and images were completely deidentified.

\section{Segmentation and Skeletonization}

High-resolution computed tomography (HRCT) scans were imported into ITK-SNAP ${ }^{12}$ (version 3.4.0). ${ }^{13}$ Using standardized techniques, the area of dehiscence was manually segmented by two authors (J. B. and C. L.) using the following protocol: Upon visualizing the dehiscence in coronally formatted images, a line was drawn across the defect with the paintbrush tool. This was repeated in each consecutive slice until bone overlying the superior semicircular canal was clearly present. Dehiscence in the sagittal plane was measured in similar fashion with focus directed to areas not originally marked in the coronal plane. Dehiscence volume was calculated using a skeletonization transform, which derives a single- pixel thick representation of the original delineation by eroding away pixels from the short axis (while preserving the long axis end points) until no more thinning is possible. It was used to calculate dehiscence volume.

\section{Statistical Analysis}

Descriptive statistics and $t$-tests were performed using the Statistical Analysis System (SAS, V9.3; SAS Institute Inc., Cary, North Carolina, United States). The mean and frequency count for continuous variables (age, sex, weight, and race) were determined. After testing for the equality of variances, either the pooled or Satterthwaite $T$ - and $p$ values were used. If the variances were unequal, as in our data, the Satterthwaite $p$ values were recorded instead of the pooled $p$ value. Statistical significance was set at a $p$ value less than 0.05 for all analyses.

\section{Results}

A total of 70 cases of SSCD were identified in 59 patients. Mean age was 50.9 years (standard deviation [SD]: 14.4, range: 21-84). Females and males comprised $66.1 \%$ $(n=39)$ and $33.9 \%(n=20)$ of the sample, respectively (approximate female to male ratio, 2:1).

An improved representation of the volumetric region that covers the defective anatomy was successfully obtained by applying a morphological skeletonization transform ${ }^{12}$ (or medial axis transform [MAT]) to the manually painted delineations. With this transform, we obtained a single-pixel thick "sheet" over the dehiscence ( - Fig. 1A-D). This representation was vetted and modified where necessary before performing further measurements. Finally, the volume of the singlepixel thick "sheet" was then computed by counting the number of nonzero image voxels encompassing the sheet and multiplying this number by the volume $\left(\mathrm{mm}^{3}\right)$ of each voxel.

Overall, the mean volume of dehiscence recorded was 0.88 $\mathrm{mm}^{3}$ (SD: 0.57 , range: $0.11-2.27$ ). Mean volume for females was $0.89 \mathrm{~mm}^{3}$ (SD: 0.60 , range: $0.11-2.27$ ) versus $0.87 \mathrm{~mm}^{3}$ (SD: 0.51, range: $0.20-1.92$ ) for males. Differences in volume by sex did not reach statistical significance.

\section{Discussion}

Since the first description of SSCD by Minor and coworkers in 1998, advancements in radiologic and vestibular testing have increased identification of the pathology. A study using dedicated $\mathrm{CT}$ of the temporal bones reported a radiologic prevalence of $4 \%,{ }^{12}$ while a large histologic study of 1,000 temporal bones demonstrated a prevalence of only $0.5 \%$. The nearly 10 -fold higher radiographic prevalence may represent an overestimation attributable to partial volume average effect. $^{12-15}$ Thus, it remains important to combine clinical, audiometric, and radiographic tools to confirm the diagnosis.

Several studies have investigated the size of dehiscence using various methods, but correlations with clinical findings remain obscure. The most common method used to measure dehiscence size involves drawing a straight line subtending the arc of maximal dehiscence in a single plane on HRCT of the temporal bone. ${ }^{2,6,9}$ Rajan et al found that the size of dehiscence 


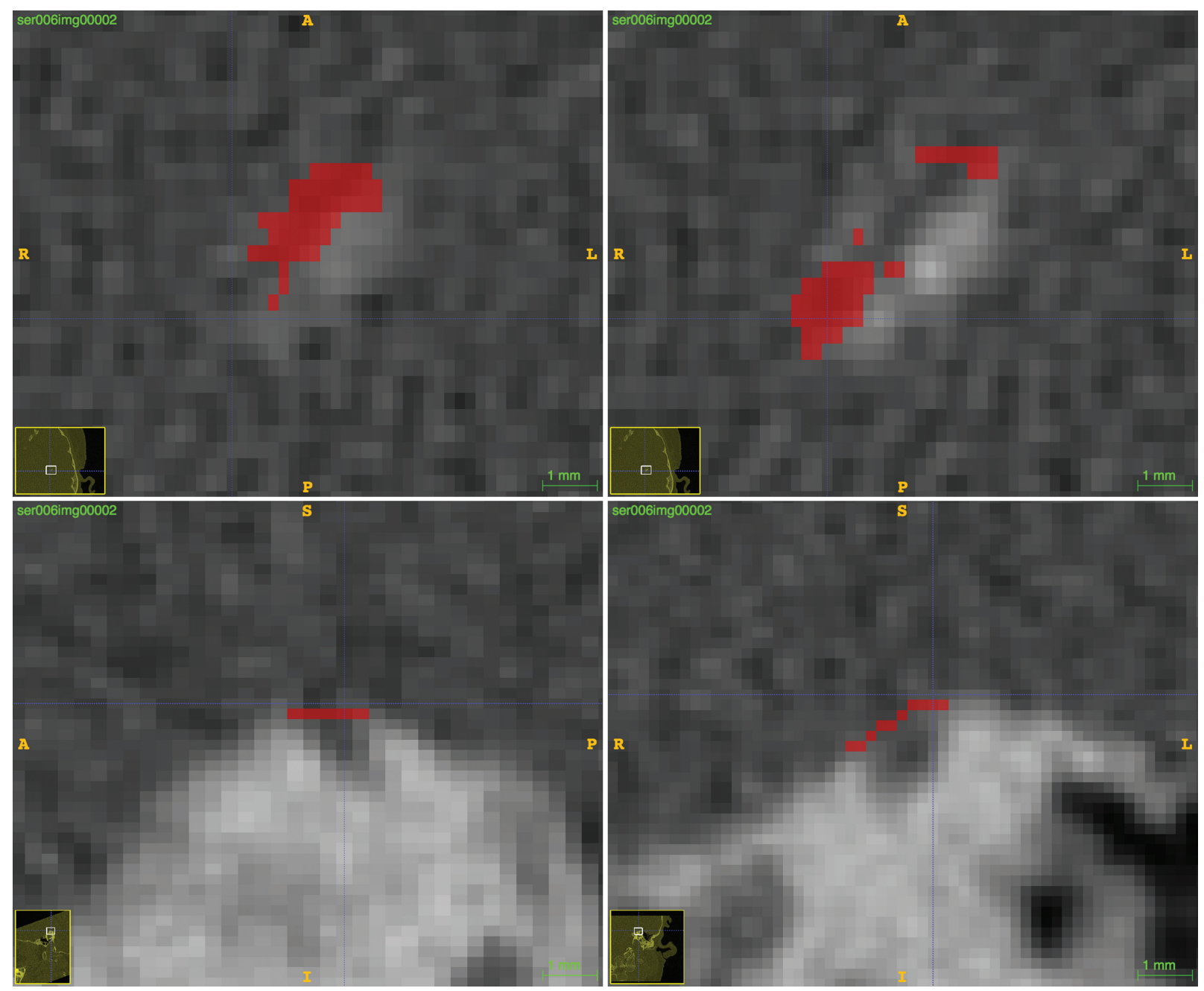

Fig. 1 ITK-SNAP (version 3.4.0; available at: http://www.itksnap.org/) visualization for contiguous axial (A and B), sagittal (C), and coronal slices (D), respectively, of superior semicircular canal dehiscence with single-pixel thick volume "sheet" (red) over the defect.

was inversely proportional to the frequency of stimuli required to provoke a vestibular response. ${ }^{2}$ The authors did not explicitly describe their technique for measuring dehiscence; however, they provided a single coronal CT image with a measurement from the most lateral edge to the most medial edge of dehiscence. Similarily, Pfammatter et al measured dehiscence using a straight line connecting the bony ends of the defect and determined that dehiscence smaller than $2.5 \mathrm{~mm}$ present with either cochlear or vestibular symptoms, while dehiscence greater than or equal to $2.5 \mathrm{~mm}$ often present with cochleovestibular symptoms. ${ }^{1}$

Correlates of dehiscence have also been investigated using more objective measures. Yuen et al used a straight line subtending the arc of the dehiscence for their measurements and determined that dehiscence size less than $3 \mathrm{~mm}$ displayed inconsistencies in $\mathrm{ABG}$ measurements, whereas size greater than $3 \mathrm{~mm}$ displayed consistent $\mathrm{ABG}$ measurements, and that dehiscence size was positively correlated with $\mathrm{ABG} .{ }^{9}$ The authors described the convenience and limitation of their method of measurement including simplicity and underestimation of the defect, respectively. The authors reported that differences between methods (straight vs. curvilinear measurements) were negligible. Nevertheless, they acknowledged the need for standardization of measurements and posited that measuring the surface area of the dehiscence would provide the most accurate representation of the pathology. Although conceptually valid, we feel that the complex shape and relatively poor resolution of dehiscence images make area calculations infeasible. Utilizing a single-pixel thick "sheet" allows rapid calculation of volume which adds depth to surface area.

Manzari et al sought to relate dehiscence size to oVEMP and cVEMP. $^{8}$ The length of dehiscence was measured using "computer-assisted methods," but specific techniques were not described. The amplitude of contralateral SSCD oVEMP n10 was shown to be strongly dependent on dehiscence size, while cVEMP demonstrated a much weaker (insignificant) correlation. That study highlights the need for standardization of measurements, which shlould facilitate multicenter investigations and ultimately validation of published findings.

Intraoperative measurements of dehiscence length have also been described in the literature and positively correlated with maximal $\mathrm{ABG}^{7}{ }^{7}$ Chien et al placed a scale adjacent to the 
area of dehiscence under the operative microscope. The authors acknowledged that the defects measured in their study were on an average larger (operative patients) and that this had the potential to affect their results. They observed a significant correlation between dehiscence length and maximum ABG. Obviously, intraoperative measurements can only be performed in patients undergoing surgical repair. Therefore, this method is neither practical nor conducive to correlative studies, that ultimately aim to identify patients with dehiscences more amenable to surgical repair.

Above, we present a convenient and cost-effective approach to measuring dehiscence size, which we believe more accurately represents the SSCD pathology. However, prudent cost-benefit analyses are needed to establish the benefits of this technique. Limitations of this study include interobserver variability. We attempted to correct for this by running multiple segmentations by two authors. The lack of clinical outcomes and comparison to techniques previously described is a glaring limit and provides minimal clinical granularity. The aim of this initial study was to present a novel method, which has the potential for standardization and can be readily applied in the clinical and investigative setting. Future studies will compare our technique to those reported in prior studies. Lastly, we will seek to validate correlations in the current literature and identify those yet to be reported using our method of measuring dehiscence volume.

\section{Conclusion}

The relationship of dehiscence size and clinical measures has been investigated with largely equivocal results. We attribute this to the lack of uniformity in measurements and misrepresentation of the defect. Advances in imaging techniques and computer-based algorithms will continue to improve the accuracy of dehiscence measurements. As methods arise and evolve, analyses relating size to clinical variables will be needed. We presented a technique, which is novel in two respects: (1) manual segmentation of dehiscence of all slices in coronal and sagittal planes and (2) calculation of dehiscence volume. To our knowledge, this is the first study to describe this method. We believe that this process captures the dehiscence more accurately, and therefore provides a stronger foundation for future correlative studies relating dehiscence size to symptomology and clinical outcomes.

\section{Disclosures}

Authors do not have any conflict of interest to disclose concerning the materials discussed in this study.

\section{Acknowledgments}

Carlito Lagman was partially supported by a Gurtin Skull Base Research Fellowship. Lawrance K. Chung was partially supported by an American Medical Association Foundation Seed Grant and an Alpha Omega Alpha Carolyn L.
Kuckein Student Research Fellowship. Isaac Yang was partially supported by a Visionary Fund Grant, an Eli and Edythe Broad Center of Regenerative Medicine and Stem Cell Research University of California, Los Angeles (UCLA) Scholars in Translational Medicine Program Award, the Jason Dessel Memorial Seed Grant, the UCLA Honberger Endowment Brain Tumor Research Seed Grant, and the STOP CANCER Research Career Development Award.

\section{References}

1 Pfammatter A, Darrouzet V, Gärtner M, et al. A superior semicircular canal dehiscence syndrome multicenter study: is there an association between size and symptoms? Otol Neurotol 2010; 31(3):447-454

2 Rajan GP, Leaper MR, Goggin L, Atlas MD, Boeddinghaus R, Eikelboom RK. The effects of superior semicircular canal dehiscence on the labyrinth: does size matter? Otol Neurotol 2008; 29(7):972-975

3 Chung LK, Ung N, Spasic M, et al. Clinical outcomes of middle fossa craniotomy for superior semicircular canal dehiscence repair. J Neurosurg 2016:1-7

4 Spasic M, Trang A, Chung LK, et al. Clinical Characteristics of Posterior and Lateral Semicircular Canal Dehiscence. J Neurol Surg B Skull Base 2015;76(6):421-425

5 Yew A, Zarinkhou G, Spasic M, Trang A, Gopen Q, Yang I. Characteristics and management of superior semicircular canal dehiscence. J Neurol Surg B Skull Base 2012;73(6):365-370

6 Martin C, Chahine P, Veyret C, Richard C, Prades JM, Pouget JF. Prospective radiological study concerning a series of patients suffering from conductive or mixed hearing loss due to superior semicircular canal dehiscence. Eur Arch Otorhinolaryngol 2009; 266(8):1175-1181

7 Chien WW, Janky K, Minor LB, Carey JP. Superior canal dehiscence size: multivariate assessment of clinical impact. Otol Neurotol 2012;33(5):810-815

8 Manzari L, Burgess AM, McGarvie LA, Curthoys IS. Ocular and cervical vestibular evoked myogenic potentials to $500 \mathrm{~Hz} f z$ boneconducted vibration in superior semicircular canal dehiscence. Ear Hear 2012;33(4):508-520

9 Yuen HW, Boeddinghaus R, Eikelboom RH, Atlas MD. The relationship between the air-bone gap and the size of superior semicircular canal dehiscence. Otolaryngol Head Neck Surg 2009;141(6):689-694

10 Chi FL, Ren DD, Dai CF. Variety of audiologic manifestations in patients with superior semicircular canal dehiscence. Otol Neurotol 2010;31(1):2-10

11 Pisano DV, Niesten ME, Merchant SN, Nakajima HH. The effect of superior semicircular canal dehiscence on intracochlear sound pressures. Audiol Neurootol 2012;17(5):338-348

12 Cloutier JF, Bélair M, Saliba I. Superior semicircular canal dehiscence: positive predictive value of high-resolution CT scanning. Eur Arch Otorhinolaryngol 2008;265(12):1455-1460

13 Belden CJ, Weg N, Minor LB, Zinreich SJ. CT evaluation of bone dehiscence of the superior semicircular canal as a cause of soundand/or pressure-induced vertigo. Radiology 2003;226(2):337-343

14 Sequeira SM, Whiting BR, Shimony JS, Vo KD, Hullar TE. Accuracy of computed tomography detection of superior canal dehiscence. Otol Neurotol 2011;32(9):1500-1505

15 Curtin HD. Superior semicircular canal dehiscence syndrome and multi-detector row CT. Radiology 2003;226(2):312-314 